\title{
Die prognostische Validität der Sekundarschulempfeh- lung in Luxemburg: Eine Gegenüberstellung von Verbleibsquoten und Leistungstestdaten
}

\section{Florian Klapproth, Sabine Krolak-Schwerdt, Sabine Glock, Matthias Böhmer und Romain Martin}

Mit der vorliegenden Studie wurde die prognostische Validität der Sekundarschulempfehlung im dritten Jahr der Sekundarstufe anhand einer repräsentativen Stichprobe luxemburgischer Schülerinnen und Schüler einer Schuljahrgangskohorte untersucht. Dazu wurden die Verbleibsquoten der Schülerinnen und Schüler auf dem jeweils empfohlenen Schulzweig ermittelt. Darüber hinaus gingen wir davon aus, dass sich eine Bewährung der Sekundarschulempfehlung auch an den individuellen Leistungen der Schülerinnen und Schüler widerspiegeln sollte. Als Mass für die Schulleistungen wurde ein standardisierter Schulleistungstest als Validierungskriterium verwendet, der zu Beginn der neunten Klasse erhoben wurde. Wir nahmen an, dass Schülerinnen und Schüler mit einer Empfehlung für einen höheren Schulzweig höhere Testwerte aufweisen sollten als diejenigen mit einer Empfehlung für einen niedrigeren Schulzweig. Darüber hinaus erwarteten wir, dass hohe Verbleibsquoten eine hohe prognostische Qualität der Empfehlung widerspiegelten. Es zeigte sich, dass die Verbleibsquoten zwar sehr hoch waren; allerdings war der Überlappungsgrad der Verteilungen des standardisierten Schulleistungstests zwischen den Schulformen ebenfalls hoch. Dieser Widerspruch zwischen unterschiedlichen Indikatoren der prognostischen Validität der Sekundarschulempfehlung deutet auf eine geringe Durchlässigkeit des luxemburgischen Schulsystems und damit generell auf die eingeschränkte Brauchbarkeit der Schätzung der prognostischen Validität über Verbleibsquoten hin.

\section{Einle itung}

Mit der vorliegenden Studie wurde die prognostische Validität der Sekundarschulempfehlung im dritten Jahr der Sekundarstufe in einer Stichprobe von Schülerinnen und Schülern aus Luxemburg untersucht. In hierarchisch gegliederten Schulsystemen werden die Schülerinnen und Schüler am Ende ihrer Grundschulzeit über die Sekundarschulempfehlung (auch Grundschulgutachten, Schullaufbahnempfehlung oder Bildungsentscheidung genannt) auf die 
unterschiedlichen Zweige der Sekundarstufe überwiesen. Den Lehrkräften der Grundschulklassen kommt im Zusammenhang mit dem Übergang ihrer Schülerinnen und Schüler die Rolle zu, eine akkurate Leistungsdiagnose und darauf aufbauend eine Prognose des schulischen Leistungspotentials eines Kindes zu erstellen. Bei der Erstellung der Sekundarschulempfehlung spielen insbesondere Zeugnisnoten eine wichtige Rolle (Arnold, Bos, Richert \& Stubbe, 2007; Ditton \& Krüsken, 2006; Ditton, Krüsken \& Schauenberg, 2005; Gröhlich \& Guill, 2009; Kristen, 2006; Lintorf, Guill \& Bos, 2008; Maaz \& Nagy, 2009; Neumann, Milek, Maaz \& Gresch, 2010; Schmitt, 2008; Schulze, Wolter \& Unger, 2009; Stahl, 2007; Stubbe \& Bos, 2008). Neben den Noten berücksichtigen Lehrkräfte in ihren Sekundarschulempfehlungen auch andere Schülermerkmale. Hierzu gehören auch Leistungen in standardisierten Schultests, so zum Beispiel in Deutschland in den Bundesländern Sachsen-Anhalt und Schleswig-Holstein (KMK, 2010), in der Schweiz im Kanton Fribourg (vgl. Baeriswyl, Wandeler, Trautwein \& Oswald, 2006) und in Luxemburg (Burton \& Martin, 2008; Reding, 2006).

Die Zuordnung der Schülerinnen und Schüler auf die unterschiedlichen Schulzweige der Sekundarstufe erfolgt auf der Basis von Kriterien, die Bestandteil der institutionellen Regularien des jeweiligen Schulsystems sind. Im Allgemeinen soll die Selektion der Schülerinnen und Schüler nach ihrer Leistungsfähigkeit erfolgen und unabhängig von leistungsfremden Merkmalen wie beispielsweise dem familiären sozioökonomischen Status sein (Trautwein \& Baeriswyl, 2007). Die Zuweisung zu den unterschiedlichen Schulzweigen soll gewährleisten, dass Schülerinnen und Schüler mit ähnlichem Leistungsniveau gemeinsam unterrichtet werden (Ansalone, 2001).

\section{Befunde zur prognostischen Validität der Sekundar- schulempfehlung}

Die prognostische Validität der Sekundarschulempfehlung wurde meist über die Bestimmung von Verbleibsquoten geschätzt. Roeder (1997) berichtet eine Studie zur prognostischen Validität von Sekundarschulempfehlungen für Hamburger Gymnasiasten. Bis zum Ende der 10. Klasse verblieben von den für das Gymnasium empfohlenen Schülerinnen und Schülern $81 \%$ auf dem Gymnasium, während von denjenigen Schülerinnen und Schülern, die keine entsprechende Empfehlung hatten, immerhin noch $43 \%$ dort erfolgreich waren. Schuchart und Weishaupt (2004) untersuchten die prognostische Validität der Sekundarschulempfehlung für niedersächsische Realschüler und Gymnasiasten am Ende der Sekundarstufe I für zwei unterschiedliche Kohorten. Bei den Gymnasiasten schlossen mit entsprechender Gymnasialempfehlung $75 \%$ (resp. $85 \%$ ) die Sekundarstufe I erfolgreich ab, während nur $28 \%$ (resp. $35 \%$ ) der Gymnasiasten ohne entsprechende Empfehlung erfolgreich waren. Bei den Realschülern mit Realschulempfehlung waren $74 \%$ (80\%) 
erfolgreich, während nur $27 \%(31 \%)$ der Realschüler ohne Empfehlung für die Realschule dort erfolgreich waren. Scharenberg, Gröhlich, Guill und Bos (2010) untersuchten im Rahmen der Kess-8-Studie (Bos \& Gröhlich, 2010) die prognostische Validität von Sekundarschulempfehlungen, die an Hamburger Grundschulen zum Ende der vierten Klasse getroffen wurden. $95 \%$ der für das Gymnasium empfohlenen Schülerinnen und Schüler verblieben nach Abschluss der 8. Klasse dort, während von den nicht empfohlenen Gymnasiasten nur noch $67 \%$ weiterhin das Gymnasium besuchten. Bei den Haupt- und Realschülern war die Diskrepanz zwischen empfohlenen und nicht empfohlenen Schülerinnen und Schülern niedriger: $89 \%$ der Schülerinnen und Schüler blieben dort mit entsprechender Empfehlung, $81 \%$ ohne Empfehlung. Tiedemann und Billmann-Mahecha (2010) ermittelten anhand eines kompletten Übertrittsjahrgangs (2004/2005) aus Niedersachsen die Verbleibsquoten in der Sekundarstufe am Ende der sechsten Klasse. Von den Schülerinnen und Schülern, die der Schulempfehlung gefolgt waren, zeigten sich über alle Schulformen betrachtet $87 \%$ erfolgreich, während 70 \% der Gymnasiasten, $61 \%$ der Realschüler und $55 \%$ der Hauptschüler ohne entsprechende Empfehlung erfolgreich waren.

Eine andere Form der Validierung der Sekundarschulempfehlung rekurriert auf den Zusammenhang zwischen der Empfehlung und späteren schulischen Leistungen. Baeriswyl, Trautwein, Wandeler und Lüdke (2009) untersuchten eine Stichprobe von insgesamt 621 Grundschülern des Schweizer Kantons Fribourg, die nach Abschluss der 6. Jahrgangsstufe in die Sekundarstufe I übertraten. Das Kriterium waren die Schulnoten in Deutsch und Mathematik am Ende der 9. Klasse. In einer Mehrebenen-Regressionsanalyse zeigte sich, dass die Vorhersagekraft der Sekundarschulempfehlung statistisch signifikant war. Allerdings reduzierte sich ihr Gewicht, wenn zusätzlich Leistungsvariablen (Testleistung und Noten) in die Regressionsgleichung aufgenommen wurden. Darüber hinaus erwiesen sich die Leistungsvariablen als vorhersagekräftiger für die Schulnoten in Klasse 9 als die Sekundarschulempfehlung.

Zusammenfassend lassen sich zwei wesentliche Befunde aus den dargestellten Untersuchungen festhalten. Erstens ist die Wahrscheinlichkeit für den Verbleib auf dem gewählten Schulzweig höher, wenn ein Kind der Sekundarschulempfehlung folgt, als wenn das Kind (bzw. dessen Eltern) eine Schulform wählt, für die es nicht empfohlen wurde. Letzteres betrifft in erster Linie Schülerinnen und Schüler, die eine höhere Schulform als empfohlen besuchen. Zweitens stellen Schulleistungen in der Grundschule zwar den stärksten Prädiktor für Schulleistungen in der Sekundarstufe dar; dennoch konnte ein eigenständiger Prognosebeitrag der Sekundarschulempfehlung auf künftige Schulleistungen festgestellt werden. Diese Befunde zur prognostischen Validität scheinen die Sekundarschulempfehlung als Selektionsinstrument zu legitimieren.

Allerdings läuft die Schätzung der prognostischen Validität der Sekundarschulempfehlung auf Grundlage von Verbleibsquoten Gefahr, den Einfluss der mangelnden Durchlässigkeit eines jeweiligen Schulsystems zu unterschätzen. 
Ein Indikator für die Durchlässigkeit ist die Häufigkeit von Schulformwechseln. In Deutschland schwankt diese zwischen $9 \%$ in Brandenburg und $24 \%$ in Schleswig-Holstein (Bellenberg, Hovestadt \& Klemm, 2004).

In der Literatur werden unterschiedliche Erweiterungen der Schätzung der prognostischen Validität der Sekundarschulempfehlung diskutiert. Zum einen können Fehlentscheidungen im Sinne einer Prognose einer für einen Schüler am «besten geeigneten» Schulform differenziert werden nach Fehlentscheidungen erster Art und Fehlentscheidungen zweiter Art (vgl. Schuchart \& Weishaupt, 2004). Eine Fehlentscheidung erster Art liegt vor, wenn der Anspruch der empfohlenen Schule das Leistungsniveau des Schülers übersteigt. Betroffen sind zum Beispiel Schülerinnen und Schüler, die eine Gymnasialempfehlung erhalten haben, aber nur einen Abschluss auf der Realschule erwerben. Umgekehrt kommt es zu einer Fehlentscheidung zweiter Art, wenn das Leistungsniveau des Schülers grösser ist als der Leistungsanspruch der empfohlenen Schule. Dies trifft zum Beispiel auf Schülerinnen und Schüler zu, die trotz einer Hauptschulempfehlung ihren Realschulabschluss erwerben. Bei Fehlentscheidungen erster Art können Schulformwechsel nach unten, schlechtere Noten, Klassenwiederholungen, unterdurchschnittliche Leistungstestwerte oder Leistungsangst resultieren. Hinweise für Fehlentscheidungen zweiter Art liefern neben dem Wechsel auf höhere Schulformen das Überspringen einer Klassenstufe, überdurchschnittliche Noten und Leistungstestwerte oder emotionale Reaktionen wie zum Beispiel Langeweile (van Ophuysen, 2006).

Aus dieser Erweiterung wird ersichtlich, dass Fehlentscheidungen auch dann vorliegen können, wenn keine Schulformwechsel erfolgt sind. Das ist insbesondere vor dem Hintergrund bemerkenswert, dass Schulformwechsel in der Regel eher selten stattfinden (Baumert, Trautwein \& Artelt, 2003; van Ophuysen, 2006). Dies mag zum einen damit zusammenhängen, dass die Schulformen häufig unterschiedliche Abschlüsse anbieten und ein Schulformwechsel oft psychische Kosten für die betroffenen Schülerinnen und Schüler nach sich zieht (van Ophuysen, 2006). Zum anderen bilden Schulformen nach Baumert und Kollegen differentielle Lern- und Entwicklungsmilieus (Baumert et al., 2003). Die zwischen den Schulformen divergente Lernentwicklung setzt sich auch im weiteren Verlauf der Sekundarstufe fort, sodass Schülerinnen und Schüler bei gleichen individuellen Voraussetzungen unterschiedlich hohe Lernfortschritte erzielen und damit langfristig unterschiedliche Chancen auf einen hohen Bildungsabschluss haben (Gröhlich, Guill, Scharenberg \& Bos, 2010).

Fehlentscheidungen im Sinne der Zuordnung eines Schülers bzw. einer Schülerin zu einer bestimmten Schulform, für die dieser oder diese nicht geeignet ist, lassen sich über die Betrachtung von Verbleibsquoten nur eingeschränkt nachweisen, da der Verbleib auf einem Schulzweig eine Eignung auch dann vorgibt, wenn sie "faktisch» nicht vorhanden ist. So kann schulische Unterforderung (als Resultat einer Fehlentscheidung zweiter Art) Konsequenzen nach sich ziehen, die auf den ersten Blick eher auf Überforderung des Schülers 
schliessen lassen. Beispielsweise kann eine längerfristige Unterforderung zu motivationalen Defiziten und zu Leistungsschwächen beitragen und den betroffenen Schüler eher als überfordert erscheinen lassen (Rimm, 1986).

Eine Möglichkeit, «ungeeignete» Schülerinnen und Schüler trotz Verbleibs auf dem gewählten Schulzweig zu erkennen, bietet die Verwendung von standardisierten Schulleistungstests. In der Kess-8-Studie (Bos \& Gröhlich, 2010) konnte gezeigt werden, dass sich die Lernleistungen unter anderem im Leseverständnis und in Mathematik auf den verschiedenen Schulformen einerseits stark unterschieden, andererseits aber auch breite Überlappungsbereiche der Leistungen bestanden. Die Überlappung der Testwerteverteilungen lässt darauf schliessen, dass beispielsweise einige Schülerinnen und Schüler, die auf der Hauptschule (oder der Realschule) erfolgreich waren, durchaus in der Lage gewesen wären, die Realschule (oder das Gymnasium) zu besuchen. Darauf weisen auch Befunde aus der LAU-7-Studie (Behörde für Schule und Berufsbildung, 2011) hin, denen zufolge auf der Basis von Ergebnissen von Schulleistungstests am Ende der 7. Klasse für knapp $14 \%$ der Schülerinnen und Schüler ein Wechsel zum Gymnasium begründbar gewesen wäre, allerdings nur $4 \%$ tatsächlich wechselten. Umgekehrt wäre bei etwa $16 \%$ der Gymnasiasten ein Wechsel zur Realschule zu empfehlen gewesen; allerdings verliessen tatsächlich nur $6 \%$ das Gymnasium.

Durch die Darstellung der Leistungsüberlappung zwischen den verschiedenen Schulformen werden indirekt auch Aussagen über die Prognosequalität der Sekundarschulempfehlung möglich. Als «Fehlentscheidungen» können solche Fälle gelten, in denen Schülerinnen und Schüler einen für die jeweilige Schulform unerwartet hohen oder niedrigen Leistungskennwert erzielen. In einer Studie zum Übergang von der Grundschule auf die weiterführenden Schulen der Sekundarstufe (van Ophuysen, 2006) wurden als Indiz für Fehlentscheidungen beim Grundschulübergang Testwerte aus Leistungstests in den Domänen Lesen und Mathematik von Schülerinnen und Schülern der 5. Klasse herangezogen. Eine Fehlentscheidung im Sinne einer Unterforderung wurde angenommen, wenn die Testleistung einer Schülerin oder eines Schülers des unteren Schulzweigs mindestens das 75\%-Perzentil der Schülerinnen und Schüler des nächst höheren Schulzweigs erreichte. Entsprechend wurde eine Fehlentscheidung im Sinne der Überforderung diagnostiziert, wenn die Testleistung einer Schülerin oder eines Schülers des höheren Schulzweigs höchstens das 25\%-Perzentil der Schülerinnen und Schüler des nächst niederen Schulzweigs erreichte (siehe auch Baumert, Trautwein \& Artelt, 2003).

Ivanov und Nikolova (2010) verwendeten im Rahmen der KESS-8-Studie ebenfalls Testwerte aus Schulleistungstests als Indikatoren für die Höhe der prognostischen Validität der Sekundarschulempfehlung. Sie postulierten eine hohe prognostische Validität, wenn die schulformspezifischen Leistungsverteilungen von Schülerinnen und Schülern mit Gymnasialempfehlung sich möglichst stark überlappen, während sich die Verteilungen von Gymnasiasten 
mit Gymnasialempfehlung und solchen ohne Gymnasialempfehlung möglichst wenig überlappen sollten.

In der vorliegenden Studie wurde für die Validierung der Sekundarschulempfehlung auf eine Stichprobe von Schülerinnen und Schülern aus einer luxemburgischen Schuljahrgangskohorte zurückgegriffen und die prognostische Validität der Sekundarschulempfehlung zu Beginn des dritten Sekundarschuljahres geschätzt. In Luxemburg folgt auf die sechsjährige Grundschulzeit der Unterricht in der Sekundarstufe. Diese ist im Wesentlichen dreigeteilt und besteht aus einem akademischen Schulzweig (Enseignement secondaire classique, ES) und zwei berufsvorbereitenden Schulzweigen (Enseignement secondaire technique, ST, und Régime préparatoire, RP). Während auf dem Enseignement secondaire technique in der Regel mittlere Schulabschlüsse erworben werden, ist das Régime préparatoire für Schülerinnen und Schüler vorgesehen, die starke Lernschwierigkeiten und Leistungsdefizite aufweisen. In Luxemburg wird die Sekundarschulempfehlung von einem Orientierungsrat getroffen (Conseil diorientation), der sich aus Schulinspektoren, dem Klassenlehrer und Lehrern der Sekundarschulen zusammensetzt. Die Empfehlung soll im Wesentlichen auf den schulischen Leistungen der Schüler in der sechsten Grundschulklasse, den Resultaten in standardisierten Leistungstests und in der Einschätzung des Arbeits- und Lernverhaltens durch die Grundschullehrkraft beruhen (Thill, 2001). Die von dem Orientierungsrat getroffene Sekundarschulempfehlung hat bindenden Charakter. Allerdings können Eltern die Entscheidung des Orientierungsrates anfechten. In diesem Fall muss sich ihr Kind einem Aufnahmeexamen stellen, welches von der aufzunehmenden Sekundarschule organisiert wird und über die Aufnahme entscheidet.

Für die Schätzung der prognostischen Validität der Sekundarschulempfehlung wurde in der vorliegenden Studie sowohl auf Verbleibsquoten als auch auf Ergebnisse aus einem standardisierten Leistungstest zurückgegriffen, den die Schülerinnen und Schüler der vorliegenden Stichprobe in der neunten Klasse durchgeführt hatten.

\section{Fragestellung und Hypothesen}

Zunächst waren wir interessiert an den Verbleibsquoten der Schülerinnen und Schüler und an dem Ausmass der Übereinstimmung zwischen der Sekundarschulempfehlung und der Schulform der Schülerinnen und Schüler in der Sekundarstufe. Je mehr Schülerinnen und Schüler auf dem empfohlenen Schulzweig verbleiben und je höher folglich die Übereinstimmung ist, als desto valider kann die Empfehlung angesehen werden. Dabei erschien uns eine Differenzierung zwischen Verbleibsquoten bei konformer Bildungsentscheidung (Schulformwahl entspricht der Sekundarschulempfehlung) und Verbleibsquoten bei diskrepanter Bildungsentscheidung (Schulformwahl entspricht nicht der Sekundarschulempfehlung) sinnvoll. Durch die Differenzierung liess sich nachvollziehen, ob eine Abweichung von der Empfehlung leistungsbedingt war (bei empfehlungskon- 
formen Schülerinnen und Schülern) oder von den Eltern oder dem Kind so gewünscht wurde (bei empfehlungsdiskrepanten Schülerinnen und Schülern). Entsprechend bisherigen Befunden aus dem deutschsprachigen Raum (Roeder, 1997; Scharenberg et al., 2010; Schuchart \& Weishaupt, 2004; Tiedemann \& Billmann-Mahecha, 2010) nahmen wir an, dass die Erfolgswahrscheinlichkeit für Schülerinnen und Schüler höher war, wenn sie der Empfehlung folgten, als wenn sie einen nicht empfohlenen Schulzweig besuchten.

Ferner gingen wir davon aus, dass sich eine Bewährung der Sekundarschulempfehlung auch an den individuellen Leistungen der Schülerinnen und Schüler in der Sekundarstufe ablesen lassen sollte. Als Mass für die Kompetenz der Schülerinnen und Schüler haben wir auf Ergebnisse eines standardisierten Schulleistungstests zurückgegriffen, der im Rahmen des nationalen School Monitoring des luxemburgischen Bildungsministeriums von der Universität Luxemburg entwickelt und zu Beginn der neunten Klasse durchgeführt worden war (Ministère de l'Éducation nationale et de la Formation professionnelle, 2010a). Dieser Test hatte gegenüber Schulnoten den Vorteil, dass die Testresultate zwischen den Schülerinnen und Schülern unterschiedlicher Schulzweige vergleichbar waren, da es auf Grund von entsprechenden Ankeritems im Rahmen einer IRT-Skalierung möglich war, die verschiedenen Testversionen zueinander in Beziehung zu setzen. Darüber hinaus war der Test so konstruiert worden, dass die massgeblichen curricularen Inhalte aller Schulzweige dort abgebildet wurden. Insbesondere vor dem Hintergrund, dass Ergebnisse aus Leistungstests in die Sekundarschulempfehlung einfliessen sollten, nahmen wir an, dass Schülerinnen und Schüler mit einer Empfehlung für den akademischen Schulzweig eher höhere Testwerte aufweisen sollten als Schülerinnen und Schüler mit einer Empfehlung für einen der berufsvorbereitenden Schulzweige. Darüber hinaus gingen wir der Frage nach, ob die Sekundarschulempfehlung über die Leistung der Schülerinnen und Schüler in der sechsten Klasse hinaus einen eigenständigen Prognosebeitrag für die Leistung derselben Schülerinnen und Schüler im dritten Jahr der Sekundarstufe liefert. Da wir davon ausgingen, dass die Leistung der Schülerinnen und Schüler in der neunten Klasse auch zum Teil über die Schulform bestimmt wird (vgl. Baeriswyl et al., 2009; Baumert et al., 2003), waren wir daran interessiert zu prüfen, inwieweit die Sekundarschulempfehlung auch bei Kontrolle der Schulform einen eigenständigen Beitrag zur Erklärung der Leistungsvarianz beitragen kann. Ergänzend wurde der Überlappungsgrad der Verteilungen von Testwerten zwischen den Schulformen betrachtet. Wenn die Sekundarschulempfehlung eine valide Einschätzung der schulischen Leistungsfähigkeit eines Kindes darstellt, dann sollten sich die Schülerinnen und Schüler hinsichtlich ihrer schulischen Kompetenzen deutlich zwischen den Schulformen der Sekundarstufe unterscheiden. Dies impliziert sowohl Unterschiede in den mittleren Leistungsscores als auch geringe Überlappungsbereiche der Verteilungen. Daher sollte die prognostische Validität der Sekundarschulempfehlung als umso höher betrachtet werden können, je stärker 
sich die mittleren Leistungsscores unterschieden und je geringer der Überlappungsgrad der Verteilungen war.

\section{Methode}

\section{St ichprobe}

Der Datensatz der vorliegenden Studie bestand aus drei Teilen. Den ersten Teil bildeten die Daten der standardisierten Schulleistungstests, welche im Rahmen der Übergangsprozedur von der Grundschule zur Sekundarschule in den 6. Klassen des Schuljahrs 2008/2009 bei allen Schülerinnen und Schülern zur Anwendung kamen. Zweitens lieferte das luxemburgische Erziehungsministerium in Zusammenarbeit mit den Lehrkräften der Grundschulen Daten über die Sekundarschulempfehlungen, die Schulnoten in den einzelnen Fächern in der sechsten Klasse der Grundschule sowie über die besuchte Schulform und Klassenstufe im Schuljahr 2011/2012. Den dritten Teil bildeten die Daten aus standardisierten Schulleistungstests, die im Rahmen des nationalen School Monitoring in Kooperation zwischen der Universität Luxemburg und dem luxemburgischen Bildungsministerium zu Beginn der 9. Klasse in der Sekundarstufe erhoben worden waren.

Die vollständige Jahrgangskohorte der luxemburgischen sechsten Klassen des Schuljahres 2008/2009 umfasste 5422 Schülerinnen und Schüler. Von dieser Grundgesamtheit konnten wir eine Stichprobe von 2980 Schülerinnen und Schülern realisieren, da nur von diesen Daten über die Sekundarschulempfehlungen, die Schulnoten und den weiteren Verbleib in der luxemburgischen Sekundarstufe vorlagen. Es gab jedoch keine Hinweise auf eine selektive Verzerrung der Stichprobe, da wesentliche Kennwerte (wie zum Beispiel Alter und Geschlecht der Schülerinnen und Schüler, die Verteilung der Nationalität der Schülerinnen und Schüler und die Verteilung der Sekundarschulempfehlungen) mit veröffentlichten Daten aus nationalen Statistiken (Ministère de l'Éducation nationale et de la Formation professionnelle, 2010b) wesentlich übereinstimmten. Tabelle 1 zeigt die Kennwerte dieser Stichprobe.

Das mittlere Alter der Schülerinnen und Schüler lag im Schuljahr 2008/2009 bei 12.6 Jahren $(S D=0.54) .327$ Schülerinnen und Schüler $(11.0 \%)$ hatten eine Klasse in der Sekundarstufe wiederholt. Etwa zwei Drittel der Schülerinnen und Schüler (64.9\%) hatten die luxemburgische Staatsbürgerschaft. Von den Schülerinnen und Schülern mit Migrationshintergrund bildeten diejenigen mit portugiesischer Herkunft die grösste Gruppe (20.0\%). Insgesamt wurden 1246 (41.8\%) Schülerinnen und Schüler der vorliegenden Stichprobe für den akademischen Schulzweig (Enseignement Secondaire Classique; ES), 1570 $(52.7 \%)$ Schülerinnen und Schüler für den höheren (Enseignement Secondaire Technique; ST) und 164 (5.5\%) Schülerinnen und Schüler für den niederen (Régime Préparatoire; RP) der berufsvorbereitenden Schulzweige empfohlen. 
Die überwiegende Mehrzahl der Schülerinnen und Schüler (97.8 \%) folgte ihrer Empfehlung.

Tabelle 1: Kennwerte der verwendeten Stichprobe

\begin{tabular}{|c|c|c|c|c|c|}
\hline \multicolumn{2}{|l|}{ Variable } & \multicolumn{2}{|r|}{$\mathrm{N}$} & \multirow[t]{2}{*}{ M } & \multirow[t]{2}{*}{ SD } \\
\hline Geschlecht & & & & & \\
\hline & weiblich & 1516 & $(50.9 \%)$ & & \\
\hline & männlich & 1464 & $(49.1 \%)$ & & \\
\hline \multicolumn{6}{|l|}{ Nationalität } \\
\hline & Luxemburgisch & 1933 & $(64.9 \%)$ & & \\
\hline & Portugiesisch & 595 & $(20.0 \%)$ & & \\
\hline & andere & 452 & $(15.1 \%)$ & & \\
\hline \multicolumn{6}{|l|}{ Sekundarschulempfehlung } \\
\hline & ES & 1246 & $(41.8 \%)$ & & \\
\hline & ST & 1570 & $(52.7 \%)$ & & \\
\hline & $\mathrm{RP}$ & 164 & $(5.5 \%)$ & & \\
\hline \multicolumn{6}{|l|}{ Schulform 7. Klasse } \\
\hline & ES & 1175 & $(39.4 \%)$ & & \\
\hline & ST & 1647 & $(55.3 \%)$ & & \\
\hline & $\mathrm{RP}$ & 158 & $(5.3 \%)$ & & \\
\hline Alter (Jahre) & & & 2980 & 12.6 & 0.54 \\
\hline Notendurchschnitt 6. Klasse & & & 2672 & 45.4 & 7.4 \\
\hline Testwert 6. Klasse & & & 2790 & 0.07 & 0.76 \\
\hline Testwert 9. Klasse & & & 2352 & 521.6 & 78.7 \\
\hline
\end{tabular}

Anmerkung: Die Kennwerte der Testwerte der 9. Klasse waren in der Eichstichprobe $M=500$ und $S D=100$. Die Kennwerte der Testwerte der 6. Klasse waren in der Eichstichprobe $M=0$ und $S D=1$.

\section{Messinstrumente}

Sekundarschulempfehlung. Die Sekundarschulempfehlung wird in Luxemburg differenziert nach den drei Sekundarschulzweigen Enseignement Secondaire Classique (ES), Enseignement Secondaire Technique (ST), und Régime Préparatoire $(\mathrm{RP})$.

Resultate der standardisierten Tests in der 6. Klasse. Im Auftrag des luxemburgischen Bildungsministeriums wird jedes Jahr in den sechsten Klassen der luxemburgischen Grundschulen die Schülerleistung in verschiedenen Fächern (Mathematik, Deutsch, Französisch) mit Hilfe standardisierter Schulleistungstests erfasst (Ministère de l'Éducation nationale et de la Formation professionnelle, 2009). Die Ausarbeitung der Aufgaben erfolgt unter Anleitung von Forschern der Universität Luxemburg durch Arbeitsgruppen, die sich aus luxemburgischen Sekundar- und Grundschullehrern zusammensetzen. 
Als Indikatoren der schulischen Kompetenz der Schülerinnen und Schüler im letzten Jahr der Grundschule lagen uns daher Testwerte von standardisierten Leistungstests vor, die in den inhaltlichen Domänen Deutsch, Französisch und Mathematik durchgeführt wurden. In den Sprachen wurden die Teilbereiche Leseverstehen, Hörverstehen und Grammatik, in Mathematik einfache Rechenaufgaben und Textaufgaben unterschieden. Die Tests wurden anhand des 2-Parameter-Logistic-Modells auf Modellkonformität überprüft und relativ zu der Gesamtpopulation z-standardisiert, sodass für jeden Kompetenzbereich (Mathematik, Deutsch, Französisch) ein Mittelwert von null und eine Standardabweichung von eins festgelegt war. Die über die jeweils unterschiedlichen inhaltlichen Teilbereiche eines Hauptfachs gemittelten standardisierten Testwerte wurden den nachfolgenden Analysen als schulische Leistungsfähigkeit zugrunde gelegt.

Zeugnisnoten 6. Klasse. Aus den Zeugnissen wurden die Noten der Hauptfächer der drei Trimester des sechsten Grundschuljahres entnommen. Die Noten lagen in Form einer Notenpunkteskala vor, die eine Abstufung der Leistung von 0 bis 60 Punkten erlaubt (Punkte unter 30 stehen für eine ungenügende Leistung). Für die Analysen wurde die über die drei Trimester der sechsten Klasse gemittelte Punktzahl in den Hauptfächern Deutsch, Französisch und Mathematik verwendet.

Resultate der standardisierten Tests in der 9. Klasse. Als Mass für die Kompetenz der Schülerinnen und Schüler in der neunten Klasse wurde auf Ergebnisse der standardisierten Schulleistungstests zurückgegriffen, die im Rahmen des nationalen School Monitoring des luxemburgischen Bildungsministeriums von der Universität Luxemburg entwickelt und zu Beginn der neunten Klasse durchgeführt worden waren (Ministère de l'Éducation nationale et de la Formation professionnelle, 2010a). Die Testitems basierten auf den Bildungsstandards, welche vom Bildungsministerium für die Sekundarstufe entwickelt wurden, sodass die massgeblichen curricularen Inhalte der Fächer Deutsch, Französisch und Mathematik aller Schulzweige dort abgebildet wurden. Es bestanden drei Testversionen, welche sich durch die allgemeine Schwierigkeit unterschieden. Durch eine entsprechend hohe Anzahl an überlappenden Ankeritems konnte jedoch eine Skalierung sämtlicher Items und sämtlicher Probanden auf derselben Skala erreicht werden. Die leichteste Testversion wurde dabei in den Régime Préparatoire Klassen eingesetzt, die mittelschwere Testversion in den Klassen des Enseignement Secondaire Technique und die anspruchsvollste Testversion in den Klassen des Enseignement Secondaire Classique. Der Mittelwert jeder Kompetenzskala wurde auf 500, die Standardabweichung auf 100 festgelegt. Für die vorliegende Untersuchung wurden aus den Testwerten der Kompetenzskalen in Deutsch, Französisch und Mathematik Mittelwerte gebildet. Die hier verwendeten Testwerte spiegeln damit eine mittlere Kompetenz der Schülerinnen und Schüler in den drei Hauptfächern wider.

Schulform und Klassenstufe im Schuljahr 2011/2012 (drittes Sekundarschuljahr). Für das Schuljahr 2011/2012 lagen Informationen über die zu diesem 
Zeitpunkt besuchte Schulform und die Klassenstufe eines Kindes der zugrunde gelegten Stichprobe vor.

\section{Datenanalysen}

Zunächst wurden für die Schülerinnen und Schüler im dritten Jahr der Sekundarstufe die Verbleibsquoten bestimmt, d. h. der Anteil derjenigen Schülerinnen und Schüler, die vom Beginn der Sekundarstufe auf dem eingangs gewählten Schulzweig geblieben sind. Die Bestimmung der Verbleibsquoten erfolgte getrennt für empfehlungskonforme und empfehlungsdiskrepante Schülerinnen und Schüler. Ausserdem erschien es sinnvoll, die Anzahl der Klassenwiederholungen zu berücksichtigen (vgl. Schuchart \& Weishaupt, 2004). Im Unterschied zum Wechsel der Schulform bleiben Schülerinnen und Schüler nach Nichtversetzung auf derselben Schulform, sie wechseln lediglich in eine andere Jahrgangs-Kohorte. Daher wurden hier die Verbleibsquoten getrennt ausgewiesen für Schülerinnen und Schüler, welche die neunte Klasse ohne Zeitverzug erreicht hatten, und für diejenigen, die im Untersuchungszeitraum eine Klasse wiederholt hatten.

Der Grad an Übereinstimmung zwischen Empfehlung und tatsächlichem Schulzweig im dritten Jahr der Sekundarstufe wurde mit Hilfe von Cohens $\kappa$ bestimmt (Cohen, 1960). Stimmen die Ausprägungen des Prädiktors (Empfehlung) mit denen des Kriteriums (Schulzweig) vollständig überein, ist $\kappa$ gleich eins. Bei zufälliger Verteilung geht $\kappa$ gegen Null. Als Daumenregel für die Interpretation der Werte von $\kappa$ schlägt Fleiss (1981) vor, dass $\kappa<.40$ eine geringe Übereinstimmung, Werte zwischen .40 und .75 eine gute Übereinstimmung und Werte oberhalb von $\kappa=.75$ eine sehr gute Übereinstimmung zwischen Prädiktor und Kriterium indizieren.

Ferner wurde der Zusammenhang zwischen der Empfehlung in der sechsten Klasse und den Testleistungen in der neunten Klasse bestimmt. Wir konnten für diese Analyse nur auf die Daten der Neuntklässler zurückgreifen und mussten die Wiederholer (also Schülerinnen und Schüler, die zum selben Zeitpunkt in der achten Klasse waren) ausschliessen, da die Leistungstests nur in den neunten Klassen durchgeführt worden waren.

Darüber hinaus wurde mittels hierarchischer linearer Regressionsanalyse geprüft, inwieweit die Sekundarschulempfehlung einen eigenständigen Aufklärungsbeitrag für schulische Leistungen zu Beginn der neunten Klasse lieferte.

Schliesslich wurde das Ausmass der prognostischen Validität über den Überlappungsgrad der Testwerteverteilungen in Klasse 9 ermittelt. Dafür wurde ein von Inman und Bradly entwickeltes Verfahren verwendet (Inman \& Bradley, 1989). 


\section{Ergebnisse}

Die prognostische Validität der Sekundarschulempfehlung auf der Grundlage von Verbleibsquoten

Zunächst wurde zwischen Verbleibsquoten bei konformer (Schulformwahl entspricht Sekundarschulempfehlung) und Verbleibsquoten bei diskrepanter (Schulformwahl steht im Widerspruch zur Sekundarschulempfehlung) Bildungsentscheidung differenziert.

Tabelle 2 zeigt die Verbleibsquoten von empfehlungskonformen Schülerinnen und Schülern, Tabelle 3 die Verbleibsquoten von empfehlungsdiskrepanten Schülerinnen und Schülern für das Schuljahr 2011/2012.

Tabelle 2: Verbleibsquoten von empfehlungskonformen Schülerinnen und Schülern mit Berücksichtigung von Klassenwiederholungen $(n=2913)$

\begin{tabular}{|l|c|c|c|}
\hline & \multicolumn{3}{|l|}{ Schulformwahl 2009/2010 } \\
\hline & ES & ST & RP \\
\hline Gesamt & $1219(100.0 \%)$ & $1541(100.0 \%)$ & $153(100.0 \%)$ \\
\hline Schulform 2011/2012 & & & \\
\hline ES ohne Wdh. & $\mathbf{1 0 6 2}(\mathbf{8 7 . 1} \%)$ & $24(1.6 \%)$ & $0(0.0 \%)$ \\
\hline ES mit Wdh. & $\mathbf{6 7}(\mathbf{5 . 5} \%)$ & $1(0.1 \%)$ & $1(0.7 \%)$ \\
\hline ST ohne Wdh. & $74(6.1 \%)$ & $\mathbf{1 2 5 8}(\mathbf{8 1 . 6} \%)$ & $38(24.8 \%)$ \\
\hline ST mit Wdh. & $16(1.3 \%)$ & $\mathbf{2 1 3}(\mathbf{1 3 . 8} \%)$ & $8(5.2 \%)$ \\
\hline RP ohne Wdh. & $0(0.0 \%)$ & $32(2.1 \%)$ & $\mathbf{1 0 6}(\mathbf{6 9 . 3} \%)$ \\
\hline RP mit Wdh. & $0(0.0 \%)$ & $13(0.8 \%)$ & $0(0.0 \%)$ \\
\hline
\end{tabular}

Anmerkung: Fett gedruckt sind die Verbleibsquoten bei Übereinstimmung zwischen Schulformwahl im Schuljahr 2009/2010 und der Schulform im Schuljahr 2011/2012. ES = Enseignement Secondaire Classique (akademischer Schulzweig), ST = Enseignement Secondaire Technique (höherer berufsvorbereitender Schulzweig), RP = Régime Préparatoire (niederer berufsvorbereitender Schulzweig).

Wie sich bei Betrachtung der Tabellen leicht erkennen lässt, verblieben die meisten Schülerinnen und Schüler auf dem Schulzweig, den sie zu Beginn der Sekundarstufe in der siebten Klasse, also im Schuljahr 2009/2010, gewählt hatten. Bei den empfehlungskonformen Schülerinnen und Schülern (Tabelle 2) waren die Verbleibsquoten auf dem akademischen Schulzweig (ES) und dem höheren berufsvorbereitenden Schulzweig (ST) deutlich höher als auf dem niederen berufsvorbereitenden Schulzweig (RP). Bei den empfehlungsdiskrepanten Schülerinnen und Schülern (Tabelle 3) waren die Verbleibsquoten auf dem jeweils selbst gewählten Schulzweig in etwa gleich hoch. 
Tabelle 3: Verbleibsquoten von empfehlungsdiskrepanten Schülerinnen und Schülern mit Berücksichtigung von Klassenwiederholungen $(n=67)$

\begin{tabular}{|l|c|c|c|}
\hline & \multicolumn{3}{|l|}{ Schulformwahl 2009/2010 } \\
\hline & ES & ST & RP \\
\hline Gesamt & $23(100.0 \%)$ & $38(100.0 \%)$ & $6(100.0 \%)$ \\
\hline Schulform $2011 / 2012$ & & & $0(0.0 \%)$ \\
\hline ES ohne Wdh. & $\mathbf{1 6}(\mathbf{6 9 . 6} \%)$ & $0(0.0 \%)$ & $0(0.0 \%)$ \\
\hline ES mit Wdh. & $\mathbf{4}(\mathbf{1 7 . 4} \%)$ & $0(0.0 \%)$ & $1(16.7 \%)$ \\
\hline ST ohne Wdh. & $2(8.7 \%)$ & $\mathbf{3 4}(\mathbf{8 9 . 5} \%)$ & $0(0 \%)$ \\
\hline ST mit Wdh. & $1(4.3 \%)$ & $\mathbf{2}(\mathbf{5 . 3} \%)$ & $\mathbf{5}(\mathbf{8 3 . 3} \%)$ \\
\hline RP ohne Wdh. & $0(0.0 \%)$ & $1(2.6 \%)$ & $\mathbf{0}(\mathbf{0 . 0} \%)$ \\
\hline RP mit Wdh. & $0(0.0 \%)$ & $1(2.6 \%)$ & \\
\hline
\end{tabular}

Anmerkung: Fett gedruckt sind die Verbleibsquoten bei Übereinstimmung zwischen Schulformwahl im Schuljahr 2009/2010 und der Schulform im Schuljahr 2011/2012. ES = Enseignement Secondaire Classique (akademischer Schulzweig), ST = Enseignement Secondaire Technique (höherer berufsvorbereitender Schulzweig), RP = Régime Préparatoire (niederer berufsvorbereitender Schulzweig).

Addiert man die Klassenwiederholer zu den Schülerinnen und Schülern, die ohne Verzögerung die neunte Klasse erreicht haben, resultierte bei den empfehlungskonformen Schülerinnen und Schülern eine Verbleibsquote von insgesamt $92.9 \%$ und bei den empfehlungsdiskrepanten Schülerinnen und Schülern eine Verbleibsquote von insgesamt $91.0 \%$. Die Verbleibsquoten waren nur unwesentlich höher, wenn die Klassenwiederholer nicht berücksichtigt werden (93.5\% resp. $93.2 \%)$.

Die Tabellen zeigen darüber hinaus, dass Wechsel auf niedrigere Schulzweige die Wechsel auf höhere Schulzweige überwogen. Insgesamt wechselten 140 Schülerinnen und Schüler auf niedere Schulzweige und 73 Schülerinnen und Schüler auf höhere Schulzweige. Im gesamten Beobachtungszeitraum wechselten folglich etwa $7 \%$ der Schülerinnen und Schüler ihren Schulzweig. Damit ist die Mobilität der Schülerinnen und Schüler (und mithin die Durchlässigkeit des Schulsystems) als gering einzustufen.

Die Daten zeigen eine leichte Überlegenheit der Lehrerempfehlung gegenüber dem davon abweichenden Elternwunsch an, wenn die Kinder eine Empfehlung für den höchsten oder den mittleren Schulzweig hatten. Allerdings sind die Verbleibsquoten für empfehlungsdiskrepante Schülerinnen und Schüler auf Grund der deutlich geringeren Fallzahl nur eingeschränkt mit den Verbleibsquoten für empfehlungskonforme Schülerinnen und Schüler vergleichbar.

Für die summarische Quantifizierung der prognostischen Validität wurde $\kappa$ berechnet. Wurden Klassenwiederholungen als Misserfolge betrachtet und folglich nicht in die Analyse aufgenommen, ergab sich für die empfehlungskonformen Schülerinnen und Schüler für die Übereinstimmung zwischen der 
Schulform in der siebten Klasse und der Schulform zwei Jahre später ein Wert von $\kappa=.91, p<.001$, und für die empfehlungsdiskrepanten Schülerinnen und Schüler war $\kappa=.90, p<.001$. Wurden hingegen die Klassenwiederholungen nicht als Misserfolge betrachtet, ergab sich für die empfehlungskonformen Schülerinnen und Schüler ein Wert von $\kappa=.89, p<.001$ und für die empfehlungsdiskrepanten Schülerinnen und Schüler war $\kappa=.87, p<.001$.

\section{Die Validierung der Sekundarschulempfehlung mit einem standardisierten Schulleistungstest}

Neben der Bestimmung der Verbleibsquoten haben wir zusätzlich den prädiktiven Stellenwert der Empfehlung im Hinblick auf individuelle Schulleistungen geprüft. Als Validierungskriterium diente dabei ein standardisierter Schulleistungstest, der zu Beginn der neunten Klasse im Schuljahr 2011/2012 in allen Sekundarschulen von Luxemburg durchgeführt worden war.

Wir waren zum einen daran interessiert festzustellen, wie eng die Sekundarschulempfehlung mit den Testleistungen in der neunten Klasse zusammenhing. Darüber hinaus gingen wir der Frage nach, ob die Empfehlung zur inkrementellen Validität beiträgt, d. h. inwieweit sie eine Vorhersage für die Testergebnisse zu leisten im Stande ist, wenn gleichzeitig Schülerleistungen aus der Grundschulzeit berücksichtigt werden. Die Schülerleistungen der Grundschule waren die über drei Trimester und die Hauptfächer Deutsch, Französisch und Mathematik gemittelten Schulnoten und die über die inhaltlichen Bereiche Deutsch, Französisch und Mathematik gemittelten Werte eines standardisierten Schulleistungstests aus der sechsten Klasse.

Wir haben zunächst über Korrelationsanalysen das Ausmass des Zusammenhangs zwischen der Sekundarschulempfehlung und anderen Prädiktoren der Schulleistung sowie dem Kriterium geschätzt. Die Korrelationsanalysen dienten dem Zweck, das Zusammenhangsmuster der Variablen zu inspizieren. Anschliessend wurden multiple Regressionsanalysen über das Kriterium «Testleistung in der 9. Klasse» durchgeführt, in welchen schrittweise nach Einbezug des Prädiktors «Sekundarschulempfehlung» weitere Prädiktoren in das Regressionsmodell aufgenommen wurden. Da nur etwa $6 \%$ aller Empfehlungen für den niedrigsten Schulzweig ausgesprochen wurden, wurde der Prädiktor «Sekundarschulempfehlung» für die anschliessenden Korrelations- und Regressionsanalysen dichotomisiert und reduzierte sich dadurch auf die beiden Stufen «Empfehlung für den akademischen Schulzweig» versus «Empfehlung für einen der beiden berufsvorbereitenden Schulzweige». Wir wollten darüber hinaus prüfen, inwieweit die Vorhersagekraft der Sekundarschulempfehlung für die spätere Leistung der Schülerinnen und Schüler abhängig war von der Schulform, die diese in der Sekundarstufe besuchten. Sowohl Sauer und Gamsjäger (1996) als auch Baeriswyl et al. (2009) konnten feststellen, dass die Prädiktionskraft in Abhängigkeit von der Schulform in der Sekundarstufe variierte. Um den Effekt der Schulform vom Effekt der Sekundarschulempfehlung trennen zu 
können, wurden hierarchische Regressionsanalysen durchgeführt, in denen die Schulform (in dichotomer Form mit den Werten «akademischer Schulzweig» versus «berufsvorbereitender Schulzweig») der Prädiktor auf Ebene 2, die Sekundarschulempfehlung, die Schulnoten und die Testwerte aus der sechsten Klasse Prädiktoren auf Ebene 1 waren. Bei den verwendeten Modellen handelte es sich um Random-Intercept-Modelle, die über restricted maximum likelihood mit dem Programmpaket HLM 6 (Raudenbush et al., 2004) geschätzt wurden. Um die relative Bedeutung der Prädiktoren abschätzen zu können, wurden alle Prädiktoren zuvor z-standardisiert, bevor sie in die Regressionsanalyse eingelesen wurden.

Tabelle 4 zeigt die Korrelationskoeffizienten für die Zusammenhänge zwischen dem Kriterium und den betrachteten Prädiktoren.

Tabelle 4: Korrelationskoeffizienten der Zusammenhänge zwischen den Prädiktoren und dem Kriterium.

\begin{tabular}{|l|c|c|c|c|c|}
\hline & $\begin{array}{c}\text { Testwert 9. } \\
\text { Klasse }\end{array}$ & $\begin{array}{c}\text { Noten- } \\
\text { durchschnitt } \\
\text { 6. Klasse }\end{array}$ & $\begin{array}{c}\text { Testwert 6. } \\
\text { Klasse }\end{array}$ & $\begin{array}{c}\text { Sekundarschul- } \\
\text { empfehlung } \\
\text { (dichotom) }\end{array}$ & $\begin{array}{c}\text { Schulform } \\
\text { in Klasse 7 }\end{array}$ \\
\hline Testwert 9. Klasse & 1 & & & & \\
\hline Notendurchschnitt 6. Klasse & $.690^{* * *}$ & 1 & & & \\
\hline Testwert 6. Klasse & $.752^{* * *}$ & $.836^{* * *}$ & 1 & & \\
\hline $\begin{array}{l}\text { Sekundarschulempfehlung } \\
\text { (dichotom) }\end{array}$ & $.610^{* * *}$ & $.760^{* * *}$ & $.747^{* * *}$ & 1 & 1 \\
\hline Schulform & $.606^{* * *}$ & $.752^{* * *}$ & $.741^{* * *}$ & $.966^{* * *}$ & 1 \\
\hline
\end{tabular}

Anmerkung: ${ }^{*}: p<.05,{ }^{* *}: p<.01,{ }^{* * *}: p<.001$. Die dichotomisierte Sekundarschulempfehlung konnte die Werte 1 («Empfehlung für den akademischen Schulzweig») oder 0 («Empfehlung für einen der beiden berufsvorbereitenden Schulzweige») annehmen. Die Schulform hatte die Werte 1 (akademischer Schulzweig) oder 0 (einer der beiden berufsvorbereitenden Schulzweige).

Offensichtlich gab es substantielle positive Zusammenhänge sowohl zwischen den einzelnen Leistungsvariablen als auch zwischen den Leistungsvariablen und der Sekundarschulempfehlung. Ähnliche, wenn auch nicht ganz so hohe Interkorrelationen zwischen Schulnoten und Testwerten berichten auch andere Autoren (z. B. Baeriswyl et al., 2009). Auffällig ist die sehr hohe Korrelation zwischen der Sekundarschulempfehlung und der gewählten Schulform, die anzeigt, dass die überwiegende Mehrzahl der Schülerinnen und Schüler ihrer Empfehlung folgten.

Wie aus Tabelle 1 ersichtlich wird, waren nicht von allen Schülerinnen und Schülern der zugrunde liegenden Stichprobe Daten aus dem Leistungstest in der neunten Klasse bekannt; auch fehlten von einigen Kindern Informationen über ihre Durchschnittsnoten in der sechsten Klasse. Fast vollständig waren jedoch die Daten zu den Leistungstestwerten aus der Grundschule. Wir haben uns gegen eine Imputation fehlender Werte aus den Leistungstests der neunten Klasse 
entschieden, da das Fehlen dieser Werte nicht zufällig zustande kam, sondern durch Klassenwiederholung bedingt war. Daher wurden die anschliessenden Regressionsanalysen auf der Grundlage von $n=2352$ Schülerinnen und Schülern durchgeführt. Die fehlenden Werte der Leistungsindizes aus der sechsten Klasse (Schulnoten und Testwerte) wurden dagegen über ein Imputationsverfahren mit Hilfe des Programms NORM, Version 2.03 (Schafer, 2000) geschätzt.

Tabelle 5 zeigt die Kennwerte der Regressionsanalysen.

Tabelle 5: Ergebnisse der hierarchischen Regressionsanalysen mit dem Kriterium "Testwert 9. Klasse».

\begin{tabular}{|c|c|c|c|c|c|c|}
\hline \multirow{2}{*}{ Prädiktor } & \multicolumn{2}{|l|}{ Modell 1} & \multicolumn{2}{|l|}{ Modell 2} & \multicolumn{2}{|c|}{ Modell 3} \\
\hline & $B$ & $S E$ & $B$ & $S E$ & $B$ & $S E$ \\
\hline Schulform & 521.72 & 18.35 & $\begin{array}{c}509.17 \\
* * *\end{array}$ & 3.75 & $\underset{* * *}{509.98}$ & 3.23 \\
\hline Empfehlung & ${ }_{* * *}^{30.75}$ & 5.10 & 0.46 & 2.10 & & \\
\hline Noten 6. Klasse & & & $\begin{array}{l}20.72 \\
*\end{array}$ & 8.86 & $\begin{array}{c}20.84 \\
*\end{array}$ & 8.87 \\
\hline Test 6. Klasse & & & $\underset{* * *}{43.51}$ & 4.01 & $\underset{* * *}{43.55}$ & 4.00 \\
\hline Deviance & \multicolumn{2}{|c|}{25975} & \multicolumn{2}{|c|}{25015} & \multicolumn{2}{|c|}{25020} \\
\hline $\begin{array}{l}\text { Anzabl der geschätzten } \\
\text { Parameter }\end{array}$ & \multicolumn{2}{|c|}{4} & \multicolumn{2}{|c|}{11} & \multicolumn{2}{|c|}{7} \\
\hline
\end{tabular}

Anmerkung: $B=$ Regressionskoeffizient, $S E=$ Standardfehler, ${ }^{*}: p<.05,{ }^{* *}: p<.01,{ }^{* * *}: p<.001$

In Modell 1 wurden nur die Sekundarschulempfehlung auf Individualebene und die Schulform auf Schulebene als Prädiktoren in das Regressionsmodell aufgenommen. Das mit Abstand grösste Gewicht erhielt die Schulform, die folglich im Wesentlichen zur Prognose der Schülerleistung in Klasse 9 beitrug. Schülerinnen und Schüler des akademischen Schulzweigs hatten im Durchschnitt höhere Leistungen in Klasse neun als Schülerinnen und Schüler der beiden berufsvorbereitenden Schulzweige. Weit geringer, aber immer noch signifikant war der Effekt der Sekundarschulempfehlung. Das Ergebnis von Modell 1 zeigt dass die Sekundarschulempfehlung trotz Kontrolle der Schulform einen substantiellen Beitrag zur Vorhersage der Schülerleistung lieferte. Schülerinnen und Schüler, die für den höchsten Schulzweig empfohlen wurden, hatten - unabhängig von der jeweils gewählten Schulform - eine tendenziell höhere Testleistung in Klasse 9 als Schülerinnen und Schüler, die für einen berufsvorbereitenden Schulzweig empfohlen waren. In Modell 2 wurden zusätzlich die Leistungsvariablen der sechsten Klasse als Prädikoren herangezogen. Sowohl die Noten als auch die Testleistung der sechsten Klasse erwiesen sich als signifikante Prädiktoren. Nach der Schulform war die Testleistung der sechsten Klasse der stärkste Prädiktor für 
die Testleistung in der neunten Klasse. Darüber hinaus zeigt Tabelle 5, dass der Sekundarschulempfehlung bei Kontrolle von Schulform und Leistungsvariablen der sechsten Klasse kein eigenständiger Anteil an der Prädiktion der Testleistung zukam. In Modell 3 wurde das Gewicht der Leistungsvariablen zur Vorhersage der Testwerte in der neunten Klasse geprüft, wenn die Sekundarschulempfehlung kein Bestandteil des Regressionsmodells war. Dabei zeigte sich, dass die Devianz sich kaum von Modell 2 unterschied, in welchem auch die Empfehlung ein Prädiktor war. Zwischen Modell 2 (Leistungsvariablen mit Empfehlung) und Modell 3 (Leistungsvariablen ohne Empfehlung) bestand praktisch kein Unterschied hinsichtlich der Devianz, während der Unterschied der Devianz zwischen Modell 1 und Modell 2 sowie zwischen Modell 1 und Modell 3 signifikant war.

\section{Die Verteilung der Testwerte in der 9. Klasse, getrennt nach Schulzweigen der Sekundarstufe}

Hierarchische Schulsysteme setzen zum einen voraus, dass Schülerinnen und Schüler im Hinblick auf ihre kognitiven Fähigkeiten und schulischen Kompetenzen kategorisiert werden können, zum anderen, dass diese jeweiligen Fähigkeiten im Rahmen von leistungshomogenen Lerngruppen optimal zur Entfaltung gelangen (vgl. van Ophuysen, 2006). Geht man davon aus, dass die schulische Kompetenz von Schülerinnen und Schülern einer bestimmten Population in der Sekundarstufe I über einen standardisierten Leistungstest gemessen werden kann und die Schülerinnen und Schüler entsprechend ihrer schulischen Kompetenz auf unterschiedlichen Schulformen unterrichtet werden, dann sollten sich bei einer hohen prognostischen Validität der Empfehlung die mittleren Leistungsscores von Schülerinnen und Schülern unterschiedlicher Schulformen stark unterscheiden, während die Verteilungen der Testwerte von Schülerinnen und Schülern unterschiedlicher Schulformen sich kaum überlappen sollten. Für die Bestimmung des Überlappungsgrades wurde die Variable „Schulform“ zunächst dadurch dichotomisiert, dass der niedrigste Schulzweig (RP) und der mittlere Schulzweig (ST) zusammengefasst wurden. Es ergaben sich folglich die beiden Stufen „akademischer Schulzweig" versus „berufsvorbereitender Schulzweig“. Für die nachfolgende Analyse lagen Testwerte von 2352 Schülern vor (vgl. Tabelle 1). Von der Analyse wurden diejenigen Schülerinnen und Schüler ausgeschlossen $(n=52)$, die nicht der Sekundarschulempfehlung folgten, da bei diesen eine Abweichung von der Empfehlung bereits zu Beginn der siebten Klasse vorlag und diese somit nicht als unmittelbar leistungsbedingt anzusehen war. Abbildung 1 zeigt die Verteilung der Testwerte, getrennt nach den Schulformen "akademischer Schulzweig" versus „berufsvorbereitender Schulzweig“. Der Mittelwert der Testleistung für Schülerinnen und Schüler des akademischen Schulzweigs ( $n=987)$ lag bei $M=580.2(S D=62.6)$, der Mittelwert der Testleistung für Schülerinnen und Schüler des berufsvorbereitenden Schulzweigs $(n=1313)$ bei $M=480.8(S D=61.0)$. Die Prüfung auf Normalverteilung 
mit dem Kolmogorov-Smirnov-Test zeigte, dass die Testwertverteilungen für Schülerinnen und Schüler beider Schulzweige nicht signifikant von der Normalverteilung abwichen.

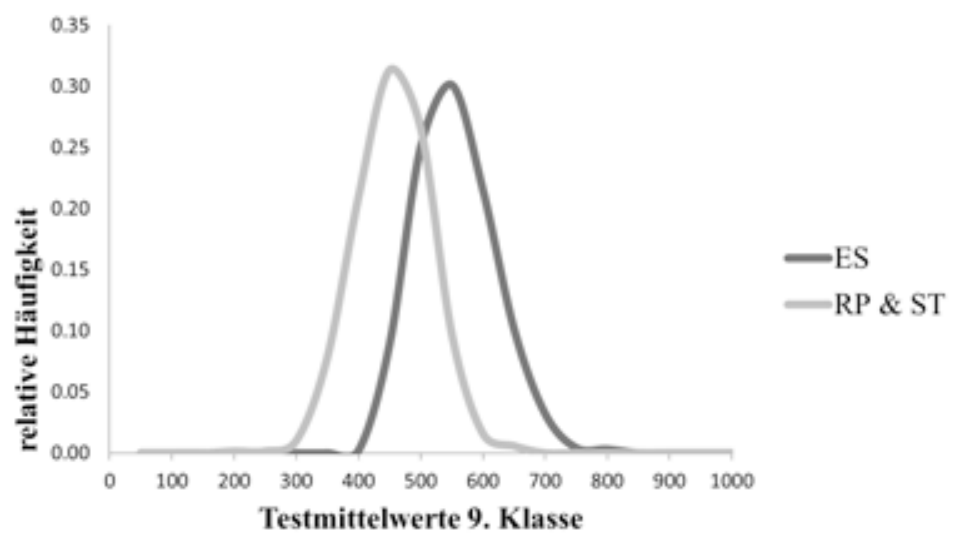

Abbildung 1: Geglättete empirische Verteilungen der Werte des standardisierten Schulleistungstests aus der neunten Klasse in Abhängigkeit von den beiden Schulformen "akademischer Schulzweig» (Ensignement secondaire classique [ES]) und "berufsvorbereitender Schulzweig»(Enseignement secondaire technique [ST] und Régime préparatoire [RP]).

Die Überlappung der beiden Verteilungen wurde mit dem Überlappungskoeffizienten nach Inman und Bradley (1989) geschätzt. Der Überlappungskoeffizient (OVL) für zwei normale Wahrscheinlichkeitsverteilungen wird bestimmt nach

$$
\mathrm{OLV}=2 \Phi\left(\frac{-\delta}{2}\right)
$$

mit

$$
\delta=\frac{|\mu 1-\mu 2|}{\sigma} .
$$

Die Verteilungsfunktion $\Phi$ beschreibt das Integral von $-\infty$ bis - $|\delta|$ über die Dichte der Standard-Normalverteilung, die Werte für $\mu_{1}$ und $\mu_{2}$ wurden über die Mittelwerte und der Wert für $\sigma$ über die gepoolte Streuung der empirischen Häufigkeitsverteilungen geschätzt. Der Überlappungsgrad für beide Verteilungen war mit OVL $=0.42$ recht hoch. Mit anderen Worten, die beiden Verteilungen teilten sich etwa $42 \%$ ihrer Fläche. 


\section{Diskussion}

In der vorliegenden Studie wurde die prognostische Validität der Sekundarschulempfehlung untersucht, die nach sechs Jahren Grundschulzeit im luxemburgischen Schulsystem erteilt wird. Die Validierungskriterien waren zum einen die Verbleibsquoten von Schülerinnen und Schülern auf den gewählten Schulzweigen zwei Jahre nach der Empfehlung, zum anderen Ergebnisse aus einem standardisierten Schulleistungstest, der zu Beginn der neunten Klasse in der Sekundarstufe I durchgeführt worden war. Wir nahmen an, dass die Verbleibsquoten für Schülerinnen und Schüler höher waren, wenn sie der Sekundarschulempfehlung folgten, als wenn sie einen nicht empfohlenen Schulzweig besuchten. Darüber hinaus erwarteten wir, dass Empfehlungen für den akademischen Schulzweig mit höheren Testwerten einhergehen sollten als Empfehlungen für einen der berufsvorbereitenden Schulzweige. Ferner interessierte uns die Frage, ob die Sekundarschulempfehlung über die Leistung der Schülerinnen und Schüler in der sechsten Klasse hinaus einen eigenständigen Prognosebeitrag für die Leistung derselben Schülerinnen und Schüler im dritten Jahr der Sekundarstufe liefert. Schliesslich nahmen wir an, dass sich hohe Verbleibsquoten in einem geringen Überlappungsgrad der Verteilungen der Testleistungen von akademischem und berufsvorbereitendem Schulzweig widerspiegeln sollten.

Die Verbleibsquote, das heisst der Anteil von Schülerinnen und Schülern, die sich zwei Jahre nach der Sekundarschulempfehlung auf demselben Schulzweig befanden wie eingangs gewählt, war für die gesamte Stichprobe mit knapp $93 \%$ ausserordentlich hoch.

Insgesamt fand sich selten eine Abweichung von der empfohlenen Schulform, nur etwa zwei Prozent der Kinder besuchten eine nicht empfohlene Schule. In den beiden oberen Schulzweigen zeigten sich nur geringfügige Unterschiede in den Verbleibsquoten zwischen empfehlungskonformen und empfehlungsdiskrepanten Schülerinnen und Schülern (wie erwartet mit leichter Überlegenheit der Quoten empfehlungskonformer Schüler). Diese Tendenz kehrte sich bei Schülerinnen und Schülern des untersten Schulzweigs um. Allerdings erlauben die geringen Fallzahlen (nur sechs empfehlungsdiskrepante Schülerinnen und Schüler im untersten Schulzweig) keine tiefere Interpretation dieses erwartungswidrigen Resultats.

Die Verbleibsquote für Schülerinnen und Schüler, welche die Sekundarstufe auf dem untersten Schulzweig begonnen hatten, war deutlich niedriger als bei Schülerinnen und Schülern der beiden höheren Schulzweige, knapp ein Drittel der Kinder wechselte im Laufe von zwei Jahren auf einen der höheren Schulzweige. Dies mag damit zusammenhängen, dass die Durchlässigkeit zwischen dem unteren und dem mittleren Schulzweig höher war als zwischen den oberen beiden Schulzweigen. Zum anderen ist es erklärtes Ziel des luxemburgischen Bildungssystems, Schülerinnen und Schüler des Régime préparatoire auf die Weiterführung des Unterrichts in den verschiedenen höheren Zweigen und Fachrichtungen der Sekundarstufe vorzubereiten. 
Hohe Verbleibsquoten stellen eine notwendige, keinesfalls aber hinreichende Bedingung für eine hohe prognostische Qualität der Empfehlung dar. In Schulsystemen mit geringen Chancen auf einen Wechsel des Schulzweigs erhöhen sich die Verbleibsquoten im Vergleich zu Schulsystemen mit höherer Durchlässigkeit. Durch die Undurchlässigkeit des Schulsystems können Kinder auf Schulzweigen unterrichtet werden, für die sie ihrer Leistung entsprechend eher ungeeignet sind. In der Kess-8-Studie (Bos \& Gröhlich, 2010) konnte zum Beispiel gezeigt werden, dass sich die Lernleistungen unter anderem im Leseverständnis und in Mathematik auf den verschiedenen Schulformen einerseits stark unterschieden, andererseits aber auch breite Überlappungsbereiche der Leistungen bestanden.

Aus diesem Grund wurden in dieser Untersuchung im Unterschied zu früheren Studien zur prognostischen Validität von Sekundarschulempfehlungen zusätzlich Kennwerte aus einem standardisierten Schulleistungstest als Validierungskriterium verwendet. Tatsächlich zeigte sich auch bei Kontrolle der Schulform ein substantieller Zusammenhang zwischen Empfehlung und Testleistung in der neunten Klasse. Darüber hinaus wiesen die Testwerteverteilungen für Schülerinnen und Schüler des akademischen Schulzweigs und Schülerinnen und Schüler der berufsvorbereitenden Schulzweige einen ausgesprochen hohen Überlappungsbereich auf. In die gleiche Richtung verwiesen die Ergebnisse aus den Regressionsanalysen. Zwar war die Sekundarschulempfehlung ein bedeutsamer Prädiktor für die Testleistung; dennoch reduzierte sich der Prognosebeitrag der Empfehlung drastisch, wenn gleichzeitig Leistungskennwerte aus der sechsten Klasse berücksichtigt wurden. Die Modellpassung konnte nicht erhöht werden, wenn zusätzlich zu den Testwerten und Schulnoten aus der sechsten Klasse die Sekundarschulempfehlung als Prädiktor in das Regressionsmodell einbezogen wurde. Folglich muss die inkrementelle Validität der Sekundarschulempfehlung in Bezug auf die Testleistung als sehr gering eingeschätzt werden.

Die Sekundarschulempfehlung in Luxemburg sagt mit hoher Wahrscheinlichkeit den Schulzweig voraus, den ein Schüler oder eine Schülerin in der neunten Klasse der Sekundarstufe besuchen wird. Der Widerspruch zwischen hohen Verbleibsquoten auf der einen Seite und dem hohen Überlappungsgrad der Testwerteverteilungen auf der anderen Seite legt allerdings die Schlussfolgerung nahe, dass diese Voraussage weniger bedingt durch die Qualität der Prognose ist, sondern auf die mangelnde Durchlässigkeit des luxemburgischen Schulsystems zurückgeht. Schülerinnen und Schüler, die durch die Sekundarschulempfehlung fehlklassifiziert wurden in dem Sinne, dass gute Schülerinnen und Schüler auf einen der berufsvorbereitenden Schulzweige und schlechte Schülerinnen und Schüler auf den akademischen Schulzweig orientiert wurden, haben offenbar nicht die Möglichkeit gehabt, auf einen ihrer Leistung besser entsprechenden Schulzweig zu wechseln.

Aufgrund der Undurchlässigkeit des Schulsystems erwiesen sich von der Empfehlung abweichende Schulformwahlen zu Beginn der Sekundarstufe als 
ebenso prädiktiv für die Schulform in der neunten Klasse wie die Empfehlungen selbst.

Eine weitere Erklärung für Leistungsdifferenzen zwischen Schülerinnen und Schülern, die Schulen unterschiedlicher Schulformen besuchen, liefert auch das Konzept der schulformspezifischen Entwicklungsmilieus (Baumert, Stanat \& Watermann, 2006). So wurde verschiedentlich berichtet, dass sich durch den Unterricht an Schulen unterschiedlicher Schulformen bei ähnlichen Grundschulleistungen unterschiedlich ausgeprägte Leistungszuwächse von Schülerinnen und Schülern feststellen liessen (Baumert et al., 2006; Trautwein et al., 2008). Schulformspezifische Effekte auf die Lernleistung lassen sich bereits nach zweijähriger Aufteilung der Schülerinnen und Schüler auf verschiedene Schulformen der Sekundarstufe I in unterschiedlichen Unterrichtsdomänen nachweisen (Bos, Bonsen \& Gröhlich, 2009) und setzen sich in den folgenden Schuljahren fort (Gröhlich, Guill, Scharenberg \& Bos, 2010). Folglich erzielen Schülerinnen und Schüler bei gleichen individuellen Voraussetzungen je nach besuchter Schulform unterschiedlich hohe Lernfortschritte und haben damit langfristig unterschiedlich hohe Chancen auf höhere Bildungsabschlüsse.

Bereits in früheren Untersuchungen wurde auf die «Haltekraft» von Schulen der Sekundarstufe, d. h. auf die Tendenz, Kinder trotz besonders guter oder besonders schlechter Leistungen auf dem eingangs gewählten Schulzweig zu belassen, hingewiesen (Scharenberg et al., 2010). Folglich stellt der Befund dieser Studie kein Phänomen des luxemburgischen Schulsystems dar, sondern scheint sich mehr oder weniger in allen Schulsystemen wiederzufinden, in denen Schülerinnen und Schüler in hierarchisch getrennten und voneinander unabhängigen Schulzweigen unterrichtet werden. Da dieser Untersuchung zufolge offenbar relativ häufig Schülerinnen und Schüler in einem Schulzweig unterrichtet wurden, der ihren Leistungen nicht entsprach, stellt sich die Frage, wie ein verhältnismässig undurchlässiges Schulsystem mit der Diskrepanz zwischen individueller Leistung (resp. Fähigkeit) und Beschulungsform umgeht. Insbesondere in Luxemburg scheinen Fehlklassifikationen von Schülerinnen und Schülern aufgrund der Sekundarschulempfehlung durch Klassenwiederholungen kompensiert zu werden. Für diese Vermutung spricht die Häufigkeit von Klassenwiederholungen in der luxemburgischen Sekundarstufe. Nach Angaben des luxemburgischen Bildungsministeriums wiederholen in der Sekundarstufe etwa $70 \%$ der Schülerinnen und Schüler der berufsvorbereitenden Schulzweige und etwa $30 \%$ der Schülerinnen und Schüler des akademischen Schulzweigs mindestens eine Klasse (Luxemburger Wort, 6. April 2011).

Darüber hinaus macht diese Studie deutlich, dass die Schätzung der prognostischen Validität der Sekundarschulempfehlung nicht bei der Bestimmung von Verbleibsquoten stehen bleiben darf, weil sonst die Gefahr besteht, die prognostische Qualität der Sekundarschulempfehlung zu überschätzen.

Eine mögliche Beschränkung der Interpretation der Ergebnisse der Regressionsanalysen in dieser Studie resultiert aus den hohen Interkorrelationen der 
Prädiktoren, die auf eine Multikollinearität hindeuten. Wenn die erklärenden Variablen hoch korreliert sind, lässt sich der Einfluss einer einzelnen Variablen schlecht isolieren, was sich in grossen Standardfehlern niederschlägt. Dadurch nimmt die Teststärke der Regressionsmodelle ab, sodass die Nullhypothese tendenziell seltener verworfen werden kann. Multikollinearität ist in erster Linie dann ein Problem, wenn das Ziel der Regressionsanalyse die Erklärung eines empirischen Zusammenhangs ist, da es schwierig bis unmöglich ist, den Einfluss eines einzelnen Prädiktors auf das Kriterium zu isolieren. Multikollinearität wird aber als unproblematisch betrachtet, wenn das Ziel in der Prognose der Ausprägung des Kriteriums besteht, da die Qualität der Prognose nicht beeinträchtigt ist (vgl. Cohen, Cohen, West \& Aiken, 2003).

Diese Studie hat gezeigt, dass die Verwendung eines standardisierten Schulleistungstests eine für die Schätzung der prognostischen Validität der Sekundarschulempfehlung sinnvolle Ergänzung darstellen kann, da der Test ermöglicht, Inkonsistenzen zwischen den Leistungen und Fähigkeiten der Kinder und ihrer jeweiligen Beschulung aufzuzeigen. Zukünftige Evaluationen der Sekundarschulempfehlung sollten darüber hinaus weitere Indikatoren schulischen Erfolgs als Validierungskriterium berücksichtigen.

\section{Literatur}

Ansalone, G. (2001). Schooling, tracking, and inequality. Journal of Children and Poverty, 7 , 33-47.

Arnold, K.-H., Bos, W., Richert, P. \& Stubbe, T. C. (2007). Schullaufbahnpräferenzen am Ende der vierten Klassenstufe. In W. Bos, S. Hornberg, K.-H. Arnold, G. Faust, L. Fried, E.-M. Lankes, K. Schwippert \& R. Valtin (Hrsg.), IGLU 2006. Lesekompetenzen von Grundschulkindern in Deutschland im internationalen Vergleich (S. 271-297). Münster: Waxmann.

Baeriswyl, F., Trautwein, U., Wandeler, C. \& Lüdke, O. (2009). Wie gut prognostizieren subjektive Lehrerempfehlungen und schulische Testleistungen beim Übertritt die Mathematik und Deutschleistung in der Sekundarstufe I? Zeitschrift für Erziehungswissenschaft, 12, 352-372.

Baeriswyl, F., Wandeler, C., Trautwein, U. \& Oswald, K. (2006). Leistungstest, Offenheit von Bildungsgängen und obligatorische Beratung der Eltern. Reduziert das Deutschfreiburger Übergangsmodell die Effekte des sozialen Hintergrunds bei Übergangsentscheidungen? Zeitschrift für Erziehungswissenschaft, 9, 373-392.

Baumert, J., Stanat, P. \& Watermann, R. (2006). Schulstruktur und die Entstehung differenzieller Lern- und Entwicklungsmilieus. In J. Baumert, P. Stanat \& R. Watermann (Hrsg.), Herkunftsbedingte Disparitäten im Bildungswesen: Differenzielle Bildungsprozesse und Probleme der Verteilungsgerechtigkeit. Vertiefende Analysen im Rahmen von PISA 2000 (S. 95-188). Wiesbaden: VS Verlag für Sozialwissenschaften.

Baumert, J., Trautwein, U. \& Artelt, C. (2003). Schulumwelten - institutionelle Bedingungen des Lehrens und Lernens. In Deutsches PISA-Konsortium (Hrsg.), PISA 2000. Ein differenzierter Blick auf die Länder der Bundesrepublik Deutschland (S. 261-331). Opladen: Leske + Budrich.

Behörde für Schule und Berufsbildung (2011). LAU - Aspekte der Lernausgangslage und der Lernentwicklung Klassenstufen 5, 7 und 9. Münster: Waxmann. 
Bellenberg, G., Hovestadt, G. \& Klemm, K. (2004). Selektivität und Durchlässigkeit im allgemein bildenden Schulsystem. Rechtliche Regelungen und Daten unter besonderer Berücksichtigung der Gleichwertigkeit von Abschlïssen. Essen: Arbeitsgruppe Bildungsforschung/ Bildungsplanung, Universität Duisburg-Essen.

Bos, W., Bonsen, M. \& Gröhlich, C. (2009). KESS 7 - Kompetenzen und Einstellungen von Schülerinnen und Schülern an Hamburger Schulen zu Beginn der Jahrgangsstufe 7. Münster: Waxmann.

Bos, W. \& Gröhlich, C. (2010). Kompetenzen und Einstellungen von Schülerinnen und Schülern am Ende der Jahrgangsstufe 8. Münster: Waxmann.

Burton, R., \& Martin, R. (2008). L'orientation scolaire au Luxembourg: "Au-delà de l'égalité des chances ... le gâchis d'un potential humain”. In R. Martin, C. Dierendonck, C. Meyers \& M. Noesen (Eds.), La place de l'école dans la société luxembourgeoise de demain (pp. 165-186). Bruxelles: De Boeck.

Cohen, J. (1960). A coefficient of agreement for nominal scales. Educational and Psychological Measurement, 20, 37-46.

Cohen, J., Cohen, P., West, S. G. \& Aiken, L. S. (2003). Applied multiple regression/correlation analysis for the behavioral sciences (3 ${ }^{\text {rd }}$ edition). Mahwah, NJ: Lawrence Erlbaum Associates.

Ditton, H. \& Krüsken, J. (2006). Der Übergang von der Grundschule in die Sekundarstufe I. Zeitschrift für Erziehungswissenschaft, 9, 348-372.

Ditton, H., Krüsken, J. \& Schauenberg, M. (2005). Bildungsungleichheit - der Beitrag von Familie und Schule. Zeitschrift für Erziehungswissenschaft, 8, 285-304.

Fleiss, J. L. (1981). Statistical methods for rates and proportions (2nd ed.). New York: Wiley.

Gröhlich, C. \& Guill, K. (2009). Wie stabil sind Bezugsgruppeneffekte der Grundschulempfehlung für die Schulformzugehörigkeit in der Sekundarstufe? Journal for Educational Research Online, 1, 154-171.

Gröhlich, C., Guill, K., Scharenberg, K. \& Bos, W. (2010). Differenzielle Lern- und Entwicklungsmilieus beim Erwerb der Lesekompetenz in den Jahrgangsstufen 7 und 8. In W. Bos \& C. Gröhlich (Hrsg.), KESS 8 - Kompetenzen und Einstellungen von Schülerinnen und Schülern am Ende der Jahrgangsstufe 8 (S. 100-106). Münster: Waxmann.

Inman, H. F., \& Bradley, E. L. (1989). The overlapping coefficient as a measure of agreement between probability distributions and point estimation of the overlap of two normal densities. Communications in Statistics - Theory and Methods, 18, 3851-3874.

Ivanov, S. \& Nikilova, R. (2010). Prognostische Validität der Schullaufbahnempfehlung aufgrund von Leistungsdaten. In W. Bos \& C. Gröhlich (Hrsg.), KESS 8. Kompetenzen und Einstellungen von Schülerinnen und Schülern - Jahrgangsstufe 8 (S. 123-140). Münster: Waxmann

KMK - Sekretariat der Ständigen Konferenz der Kultusminister der Länder in der Bundesrepublik Deutschland (2010). Übergang von der Grundschule in Schulen des Sekundarbereichs I und Förderung, Beobachtung und Orientierung in den Jahrgangsstufen 5 und 6 (sog. Orientierungsstufe). Bonn: Autor. Zugriff am 23.08.11 unter http://www.kmk. org/fileadmin/veroeffentlichungen_beschluesse/2010/2010_10_18-Uebergang-Grundschule-S_eI1-Orientierungsstufe.pdf

Kristen, C. (2006). Ethnische Diskriminierung in der Grundschule? Die Vergabe von Noten und Bildungsempfehlungen. Kölner Zeitschrift für Soziologie und Sozialpsychologie, 58, 79-97.

Lintorf, K., Guill, K. \& Bos, W. (2008). Grundschulübergang in Nordrhein-Westfalen: Einflussfaktoren auf das Lehrerurteil im Prognoseunterricht. In E.-M. Lankes (Hrsg.), Pädagogische Professionalität als Gegenstand empirischer Forschung (S. 99-110). Münster: Waxmann.

Luxemburger Wort (6. April 2011). Viele «Sitzenbleiber» im Lycée technique. Zugriff am 30.8.2012 unter http://www.wort.lu/de/view/viele-sitzenbleiber-im-lycee-technique-4f61c177e4b0860580aa495c 
Maaz, K. \& Nagy, G. (2009). Der Übergang von der Grundschule in die weiterführenden Schulen des Sekundarschulsystems: Definition, Spezifikation und Quantifizierung primärer und sekundärer Herkunftseffekte. Zeitschrift für Erziehungswissenschaft, 12, 153-182.

Ministère de l'Education nationale et de la Formation professionnelle (2009). Les épreuves standardisées à la fin du $4^{e}$ cycle de l'enseignement fondamental (Die standardisierten Prüfungen am Ende des 4. Zyklus der Grundschule). Luxembourg: MENFP.

Ministère de l'Education nationale et de la Formation professionnelle (2010a). Nationaler Bericht Epreuves Standardisées (EpStan). Schuljahr 2009/2010. Luxembourg: MENFP.

Ministère de l'Education nationale et de la Formation professionnelle (2010b). Les chiffres clés de l'éducation nationale - statistiques et indicateurs 2008-2009. Luxembourg: MENFP.

Neumann, M., Milek, A., Maaz, K. \& Gresch, C. (2010). Zum Einfluss der Klassenzusammensetzung auf den Übergang von der Grundschule in die weiterführenden Schulen. In Bundesministerium für Bildung und Forschung, K. Maaz, J. Baumert, C. Gresch \& N. McElvany (Hrsg.), Der Übergang von der Grundschule in die weiterführende Schule: Leistungsgerechtigkeit und regionale, soziale und ethnisch-kulturelle Disparitäten, Bildungsforschung Band 34 (S. 229-252). Bonn, Berlin: BMBF.

Raudenbush, S., Bryk, A., Cheong, Y. F., Congdon, R., \& Toit, M. (2004). HLM 6. Hierarchical linear and nonlinear modelling. Lincolnwood, IL: Scientific Software International.

Reding, P. (2006). Le passage primaire-postprimaire: Analyse de la procédure d'orientation. Luxembourg: MENFP.

Rimm, S. B. (1986). Underachievement syndrome: Causes and cures. Watertown, WI: Apple Publishers.

Roeder, P. M. (1997). Entwicklung vor, während und nach der Grundschulzeit: Literaturüberblick über den Einfluss der Grundschulzeit auf die Entwicklung in der Sekundarschule. In F. E. Weinert \& A. Helmke (Hrsg.), Entwicklungen im Grundschulalter (S. 405-421). Weinheim: Beltz/PVU.

Sauer, J. \& Gamsjäger, E. (1996). Ist Schulerfolg vorhersagbar? Die Determinanten der Grundschulleistung und ihr prognostischer Wert für den Sekundarschulerfolg. Göttingen: Hogrefe.

Schafer, J. L. (2000). NORM 2.03 for Windows 95/98/NT [Computer Software].

Scharenberg, K., Gröhlich, C., Guill, K. \& Bos, W. (2010). Schulformwechsel und prognostische Validität der Schullaufbahnempfehlung in der Jahrgangsstufe 4. In W. Bos \& C. Gröhlich (Hrsg.), KESS 8. Kompetenzen und Einstellungen von Schülerinnen und Schülern - Jahrgangsstufe 8 (S. 115-123). Münster: Waxmann

Schmitt, M. (2008). Die Bedeutung von sozialer Herkunft und bundeslandspezifischen Übergangsregelungen für die Grundschulempfehlung. In E.-M. Lankes (Hrsg.), Pädagogische Professionalität als Gegenstand empirischer Forschung (S. 111-121). Münster: Waxmann.

Schrader, F.-W. (2006). Diagnostische Kompetenz von Eltern und Lehrern. In D. H. Rost (Hrsg.), Handwörterbuch Pädagogische Psychologie (3. überarb. u. erw. Aufl ., S. 95-100). Weinheim: Psychologie Verlags Union.

Schuchart, C. \& Weishaupt, H. (2004). Die prognostische Qualität der Übergangsempfehlungen der niedersächsischen Orientierungsstufe. Zeitschrift für Pädagogik, 50, 882-902.

Schulze, A., Wolter, F. \& Unger, R. (2009). Bildungschancen von Grundschülern: Die Bedeutung des Klassen- und Schulkontextes am Übergang auf die Sekundarstufe I. Kölner Zeitschrift für Soziologie und Sozialpsychologie, 61, 411-435.

Stahl, N. (2007). Schülerwahrnehmung und -beurteilung durch Lehrkräfte. In H. Ditton (Hrsg.), Kompetenzaufbau und Laufbahnen im Schulsystem. Ergebnisse einer Längsschnittuntersuchung an Grundschulen (S. 171-198). Münster: Waxmann.

Stubbe, T. C. \& Bos, W. (2008). Schullaufbahnempfehlungen von Lehrkräften und Schullaufbahnentscheidungen von Eltern am Ende der vierten Jahrgangsstufe. Empirische Pädagogik, 22, 49-63. 
Thill, M. (2001). La nouvelle procédure de passage de l'enseignement primaire (public et privé) vers l'enseignement secondaire et secondaire technique: Résultats des conseils d'orientation et des procédures de recours. [Der neue Übergang von der Grundschule in die Sekundarschule und technische Sekundarschule: Ergebnisse des Orientierungsrats und der Beschwerdeprozeduren]. Luxembourg: MENFP.

Tiedemann, J. \& Billmann-Mahecha, E. (2010). Wie erfolgreich sind Gymnasiasten ohne Gymnasialempfehlung? Die Kluft zwischen Schullaufbahnempfehlung und Schulformwahl der Eltern. Zeitschrift für Erziehungswissenschaft, 13, 649-660.

Trautwein, U., \& Baeriswyl, F. (2007). Wenn leistungsstarke Klassenkameraden ein Nachteil sind: Referenzgruppeneffekte bei Übertrittsentscheidungen. Zeitschrift für PädagogischePsychologie, 21, 119-133.

van Ophuysen, S. (2006). Zur Analyse der prognostischen Qualität von Schullaufbahnempfehlungen. In W. Bos, H. G. Holtappels, H. Pfeiffer, H. G. Rolff \& R. Schulz-Zander (Hrsg.), Jahrbuch der Schulentwicklung, 14 (S. 49-79). Weinheim: Juventa.

Schlagworte: Sekundarschulempfehlung, prognostische Validität, Verbleibsquoten, Leistungstest, Luxemburg

\section{La validité pronostique de la procédure d'orientation au secondaire: une comparaison entre les filières fréquentées par les élèves et leurs résultats aux épreuves standardisées}

\section{Résumé}

Cette recherche examine la validité pronostique de la procédure d'orientation au secondaire d'un échantillon représentatif d'élèves luxembourgeois de troisième année secondaire. Pour ce faire, elle prend en compte le nombre d'élèves en fonction de chaque filière de formation, en considérant que la validité de la procédure d'orientation se refléterait dans les résultats obtenus individuellement à une épreuve standardisée réalisée en début de $9^{\mathrm{e}}$ année. L'hypothèse est que les résultats des élèves orientés dans des filières à exigences élevées seraient meilleurs que les résultats des élèves orientés dans des filières à moindre exigence. De ce point de vue, un grand nombre d'élèves encore scolarisés dans chacune des filières confirmerait la qualité de la procédure d'orientation. Les résultats révèlent qu'un très grand nombre d'élèves est toujours scolarisé dans la même filière mais que, par ailleurs, le recoupement des résultats entre les filières est important. Cette contradiction entre différents indicateurs démontre que le système scolaire luxembourgeois n'est pas perméable, ce qui réduit considérablement la portée d'une mesure de la validité pronostique de la procédure d'orientation sur la base du nombre d'élèves restant dans chaque filière.

Mots-clés: Procédure d'orientation primaire secondaire, validité pronostique, filières, épreuves standardisées, Luxembourg 


\title{
II valore predittivo di una procedura di orientamento nel passaggio alla scuola secondaria in Lussemburgo. Un confronto tra prosecuzione nella filiera e risultati scolastici.
}

\begin{abstract}
Riassunto
Oggetto di questa ricerca è un esame della validità predittiva della procedura di orientamento nel passaggio alla scuola secondaria tramite di un campione rappresentativo di allievi del Lussemburgo nel terzo anno del secondario II. È stato calcolato il numero di allievi che frequentano ancora la specializzazione che avevano scelto tre anni prima. Inoltre, si suppone che la validità predittiva dovrebbe riflettersi nei risultati scolastici misurati attraverso prove standardizzate svolte all'inizio della 9a classe. La nostra ipotesi è che i risultati degli allievi orientati verso filiere ad alte aspettative saranno migliori di quelli orientati verso filiere con minori aspettative. Se la maggior parte degli allievi frequenta ancora la specializzazione sulla quale si era orientato, questo potrà essere considerato come indice che la decisione di orientamento è stata valida. Lo studio dimostra che il numero di allievi che frequentano ancora la specializzazione sulla quale sono stati orientati è molto elevato, ma che la distribuzione dei risultati scolastici nelle due filiere si sono molto avvicinate. Questa contraddizione tra i due indicatori indica che il sistema scolastico lussemburghese non è permeabile, il che implica che una stima della validità predittrice basato unicamente sul numero di allievi che resta nella propria specializzazione non è affidabile.
\end{abstract}

Parole chiave: Orientamento per la transizione alla scuola secondaria, validità predittiva, tassi di continuazione, test di profitto, Lussemburgo

\section{The predictive validity of school placement decisions in Luxembourg: A comparison between track continuation rates and test scores.}

\section{Summary}

Within this study, the predictive validity of school placement decisions was assessed after three years of schooling in Luxembourgish secondary school. For this purpose, continuation rates of the students within each school track, as well as individual scores on standardized scholastic achievement testswere collected at the beginning of ninth grade. We assumed that students oriented to the highest expectations track in secondary school should achieve higher test scores than students who were recommended for one of the lower tracks. Moreover, it was expected that a high number of students still continuing within the track they had been assigned to, should indicate a high validity of the initial school placement decisions. However, results show that although continuation rates 
were high, the degree of overlap between the test-score distributions related to the different tracks was also high. The contradiction between these different indicators of the predictive validity of school placement decisions suggests that the permeability of the Luxembourgish secondary school is rather low. Therefore, the use of continuation rates as the only measure of predictive validity seems to be inappropriate.

Keywords: School placement decision, predictive validity, track continuation rates, achievement test, Luxembourg 
